\title{
What mechanisms coordinate leg movement in walking arthropods?
}

\author{
Holk Cruse
}

The construction of artificial walking machines has been a challenging task for engineers for several centuries. Advances in computer technology have stimulated this research in the past two decades, and enormous progress has been made, particularly in recent years. Nevertheless, in comparing the walk of a six-legged robot with the walk of an insect, the immense differences are immediately obvious. The walking of an animal is much more versatile, and seems to be more effective and elegant. Thus it is useful to consider the corresponding biological mechanisms in order to apply these or similar mechanisms to the control of walking legs in machines. Until recently, little information on the biological control mechanisms has been available; this paper summarizes recent developments.

Studies are beginning to be carried out on the neuronal control of the walking system ${ }^{1}$, mainly using insects and crustaceans.

\section{Control of the individual leg}

The results described here mainly concern the coupling mechanisms between legs, i.e. those mechanisms that produce a proper coordination of the walking legs even when walking is disturbed. However, it is helpful first to consider how the movement of an individual leg is controlled. The mechano-neuronal system that produces this movement might be called the 'leg-movement pattem generator'. To avoid a possible misunderstanding, it should be stressed that it is completely open whether this pattern generator contains an endogenous central pattern generator.

For simplicity, only forward walking will be discussed. The cyclic movement of a walking leg consists of two parts (Fig. 1A), the power stroke (also stance phase or support phase) and the return stroke (also swing phase or recovery phase). During the power stroke, the leg is on the ground where it can support and propel the body. In a forward-walking animal, this corresponds to a retraction movement of the leg. During the return stroke, the leg is lifted off the ground and swung to the starting position for the next power stroke. In forward walking this corresponds to a protraction movement of the leg. In the stick insect, which has been investigated in detail, the movement of the leg during both the power stroke and the return stroke is controlled by at least one servo-system, in this case, a system using negative feedback. The servo-system regulates a parameter corresponding to the velocity of the $\mathrm{leg}^{2-5}$. This was later shown to be true even for a single joint ${ }^{6,7}$. With respect to the temporal pattern of stepping, the critical question is how the pattern generator decides whether or not the transition from one mode (power or return stroke) to the other should be made. Experimental results have shown that for insects the most critical point is the transition from power stroke to return stroke. This seems to be intuitively clear as this transition terminates the support function of the leg; if performed at the wrong moment the animal may be left unsupported, which may be hazardous. Nevertheless, other solutions are also possible (see below). Three parameters influence this transition position, load, and phase in the step cycle of the other leg. The first two depend on proprioceptive information describing the state of the leg itself; these
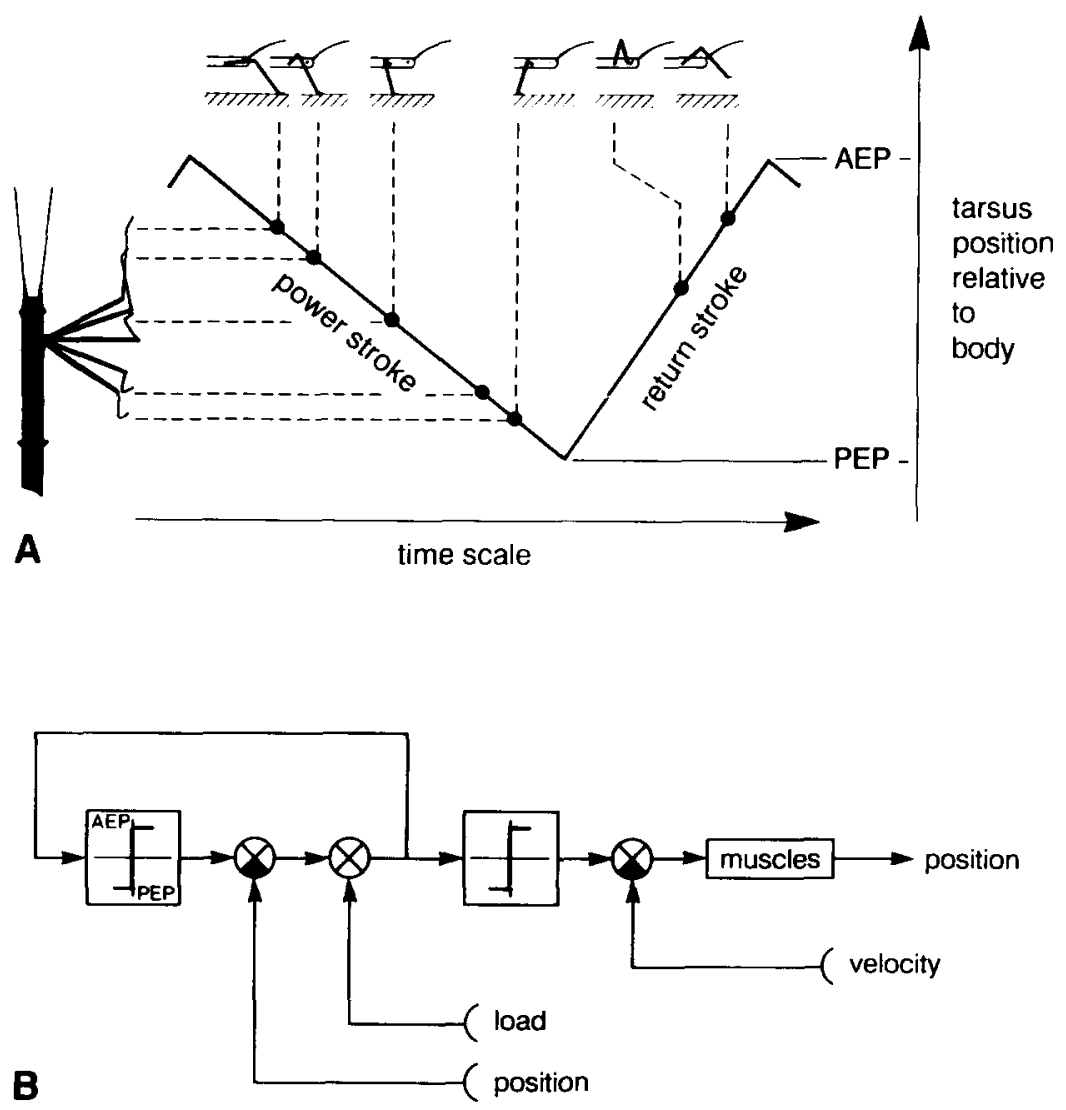

Fig. 1. (A) The cyclic movement of a leg consists of the power stroke, during which the leg supports and propels the body, and the return stroke, during which the leg is swung back to the initial position. In forward walking the latter is called the anterior extreme position (AEP). The power stroke, which corresponds to a retraction movement of the leg, ends at the posterior extreme position (PEP). (Position of the leg always means the position of the leg tip relative to the body.) (B) Circuit model of the movement of a leg. The lefthand part represents a hierarchically upper-level circuit that decides which of the two states, power stroke or return stroke, should be adopted by the system. The left relay characteristic produces the two alternative target positions, AEP or PEP, when its input value is positive or negative, respectively. The value of the target position is compared with the actual leg position. The result is also affected by signals from load-sensitive organs. The output of the decision circuit is considered as reference input for the second, lower-level circuit (right), a velocity-controlling feedback system. A positive output value of the decision system corresponds to a movement in the anterior direction (return stroke) and a negative value to a movement in the posterior direction (power stroke). 

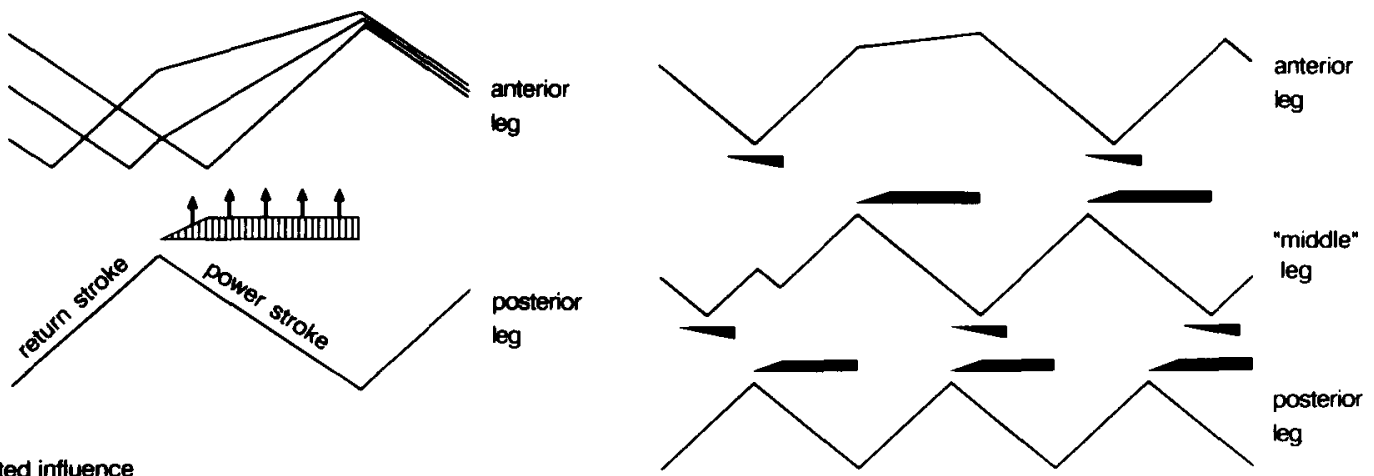

A rostrally directed influence

prolongs return stroke of anterior leg

C
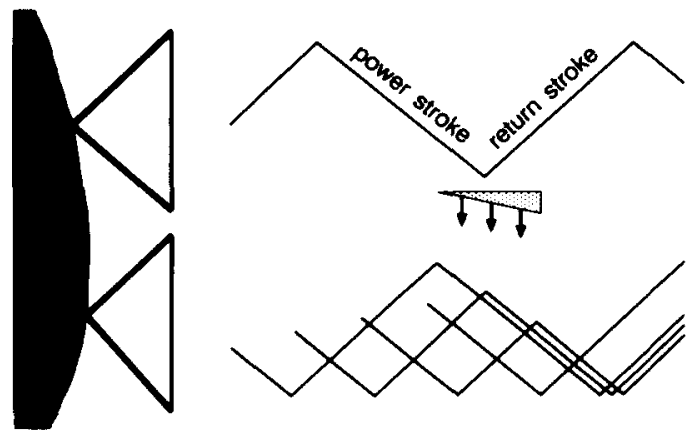

anterior leg

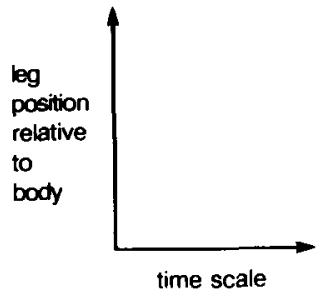

B caudally directed influence

shortens return stroke of posterior leg

Fig. 2. (A, B) Coordination between the ipsilateral legs of a crayfish. The upper traces show the anterior leg. Each schema is drawn as if only one of the two coordinating mechanisms existed. In each case the influencing leg is drawn only once. For the influenced leg several traces are presented to show the effect of the coordinating mechanism. The duration and intensity of the influences are roughly indicated by the length and thickness of the wedges, respectively. (A) The rostrally directed influence is active during the power stroke of the posterior leg. It prolongs the return stroke of the anterior leg and can also decrease the speed of the limb movement. (B) The caudally directed excitatory influence is active at the end of the power stroke and the beginning of the return stroke of the anterior leg. It 'excites' the start of a power stroke in the controlled, posterior leg. (C) Coordination between three ipsilateral legs when both forward and caudally directed influences are active. The top trace shows the most anterior leg. The 'middle' leg obtains a signal from the anterior leg which excites a power stroke. At the same time the middle leg obtains an influence from its posterior neighbour to perform a return stroke. As the latter signal is initially weak, the signal from the anterior leg 'wins' until this influence ceases and the rostrally directed influence dominates. (Modified from Ref. 13.)

peripheral influences ensure that the leg only lifts off the ground when its position relative to the body is far enough to the rear and that it only lifts off when the load under which it stands is small enough ${ }^{8,9}$.

These results can be summarized by the circuit model shown in Fig. $1 \mathrm{~B}^{10}$. The first part of the model is a hierarchically superior circuit that decides which of the two states, power stroke or return stroke, the system should adopt. The relay characteristic produces the two alternative target positions AEP and PEP (anterior and posterior extreme positions) when its input value is positive or negative, respectively. The value of the target position is compared with the actual leg position. The result is also affected by signals from load-sensitive organs. The output of the decision circuit is considered as reference input for the second, inferior circuit, a velocity-controlling feedback system. A positive output value of the decision circuit corresponds to a movement in the anterior direction (positive velocity, return stroke) and a negative value to movement in the posterior direction (negative velocity, power stroke). Results reported by Bässler ${ }^{7}$ support the assumption that load-sensitive organs also affect the system on the lower level, which is however not shown in Fig. 1B.
The model could easily be reformulated in neuronal terms. The decision part can, for example, be represented by a bistable circuit, which consists of two neurons that reciprocally inhibit each other, as described by Land ${ }^{11}$ and Bässler ${ }^{12}$.

\section{Coordination between legs}

The third parameter is of major importance here because it depends on coordinating influences: the transition from power stroke to return stroke of one leg also depends on the relative timing or the phase of the leg's movement to that of the other legs. As these influences do not originate in the leg's own periphery but have to be mediated via neuronal pathways from the pattern generators of other legs, they might be called 'internal' influences. In addition, direct peripheral influences from the sense organs of neighbouring legs might exist which are not involved in the pattern generators of these legs. Not only are coordinating mechanisms known that influence the power-return stroke transition, but also mechanisms that influence the return-power stroke transition at the AEP and mechanisms that influence the strength of the motor output during the power or the return stroke. The experimental data show no essential differences in the 
peripheral influences within a leg among insects, crustaceans, and even mammals. In contrast, comparative investigations have shown that the coordinating mechanisms are quite different for crustaceans ${ }^{13}$, insects ${ }^{14,15}$, and mammals ${ }^{16}$. The latter, however, are not considered here, and we concentrate on results obtained for the two most thoroughly investigated species, the crayfish (Astacus leptodactylus) and the stick insect (Carausius morosus).

\section{Ipsilateral coupling}

As a common feature it can be stated that in arthropods, coupling between ipsilateral legs, i.e. legs on the same side of the body, is generally stronger than coupling between contralateral legs, i.e. legs on opposite sides of the body. Therefore ipsilateral mechanisms are easier to investigate and will be treated first.

To begin with the crayfish, two coordinating mechanisms are found acting between neighbouring ipsilateral legs ${ }^{13}$. These two influences are schematically presented in Fig. 2. The two traces represent the movement of the two legs. Several phase situations are plotted in each sketch. They might be produced by a disturbance of the normal walk due, for example, to an experimental interference. One coordinating mechanism is only rostrally directed. This is illustrated in Fig. 2A: as long as the posterior leg performs a power stroke, the anterior leg has to perform or continue a return stroke. In addition, the velocity of the movement during the return stroke is also decreased to some extent. Thus the return stroke can be prolonged so that normal coordination is regained in the next step. The horizontal striped bar indicates the time during which this mechanism is active. The intensity of the influence is roughly indicated by the thickness of the bar. The second influence, illustrated in Fig. 2B, is caudally directed. When the anterior leg is near the end of its power stroke or at the beginning of its return stroke, an influence with increasing intensity has the effect of ending the return stroke and beginning the power stroke of the posterior leg, thus shortening the return stroke of this leg. As indicated by the stippled wedge, this influence ends abruptly about $200 \mathrm{~ms}$ after the beginning of the return stroke of the anterior leg. Both coordinating mechanisms influence the transition from return stroke to power stroke.

A crayfish has four ipsilateral legs. Thus two of these legs have both an anterior and a posterior neighbour. These 'middle' legs might therefore obtain both influences which, depending on their temporal relation, could superimpose. When the middle leg obtains the signal from the anterior leg at the beginning of the power stroke of the posterior leg, this can produce a long return stroke, interrupted by a short power stroke, in the middle leg (see Fig. 2C). So in this situation the caudally directed influence is somewhat stronger. It is the transition from power to return stroke that is affected, which means that the PEP is shifted to a more rostral position. In any case both mechanisms act together so that an incorrect phase relation between two legs is immediately corrected within the following step. Although for convenience the mechanisms are described as influencing the transition between the two states, this example shows that the influence could also be described as facilitating one of the two states. By means of a model calculation it can be shown that each mechanism alone would be sufficient to produce a coordinated walk. The connections can therefore be considered redundant.

\section{Contralateral coupling}

To couple the contralateral legs of the same segment, the crayfish uses a mechanism that closely resembles the ipsilateral, caudally directed influence (Müller, U., unpublished observations). In contrast to

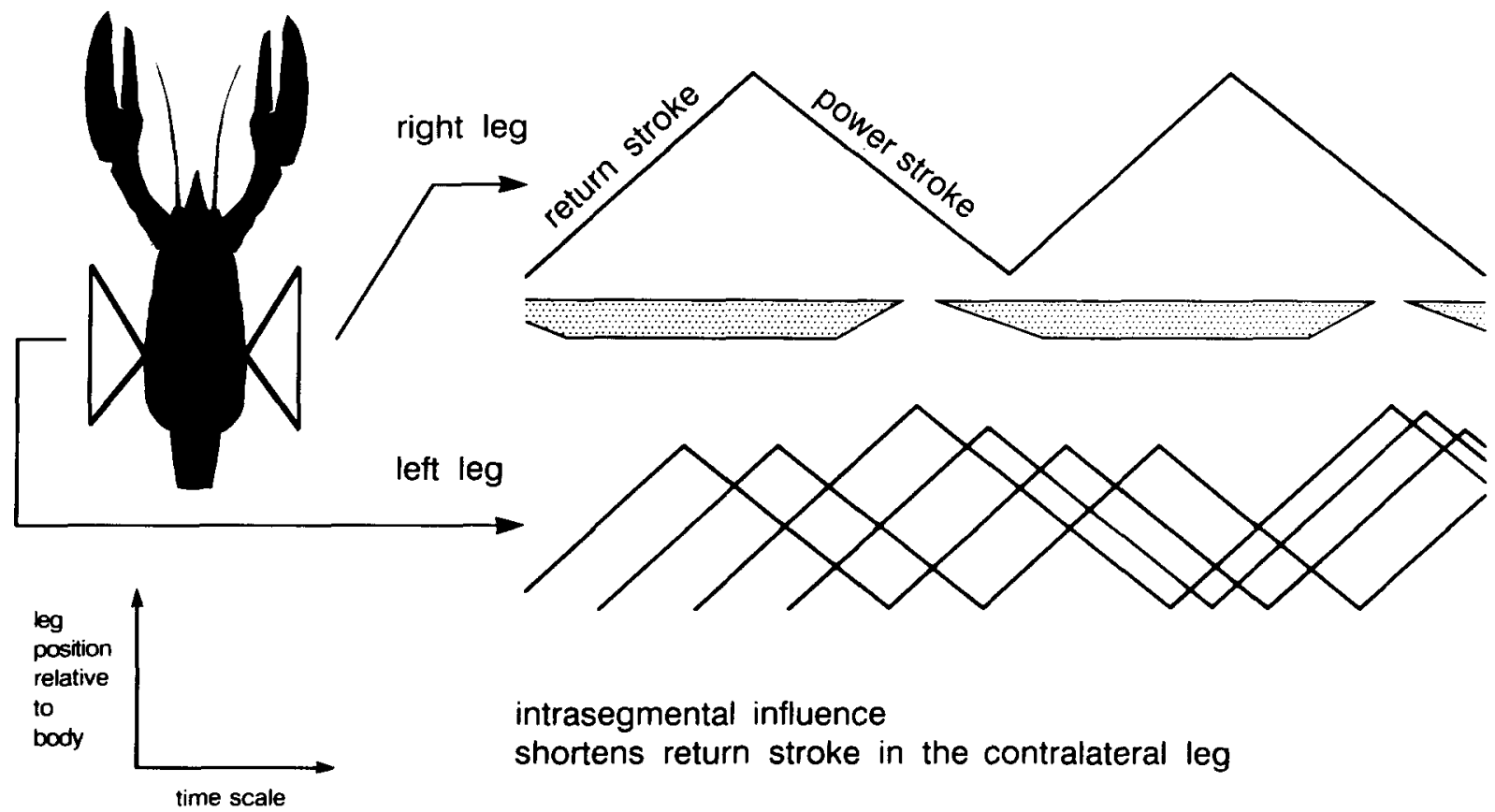

Fig. 3. Influences between two contralateral legs of a crayfish. The influences are assumed here to act only in one direction, from right to left. As in the ipsilateral, caudally directed coupling mechanism (Fig. 2B), the controlled leg is influenced to start a power stroke. The influence continues for a much longer part of the step period than in the ipsilateral case but the influence is weaker. Thus several steps could be necessary to retain the normal coordination, and relative coordination might eventually be observed (Müller, U., unpublished observations). 


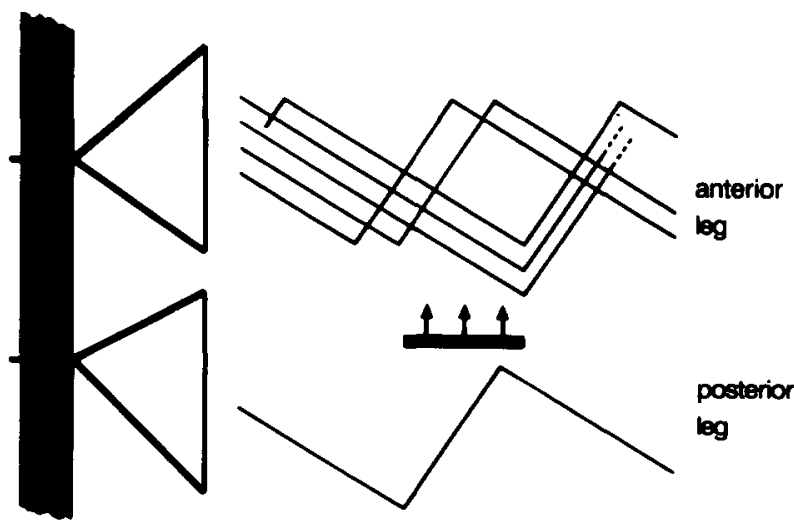

A

rostrally directed influence

inhibits start of return stroke in the anterior leg

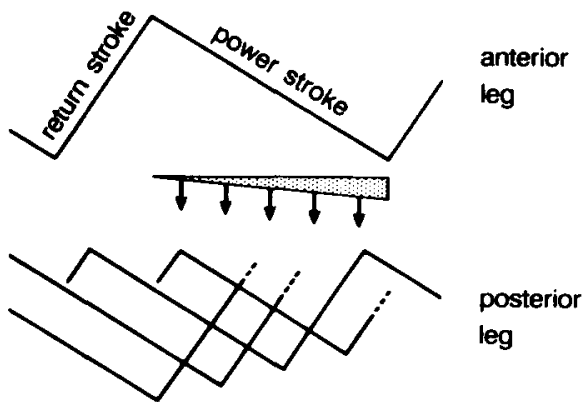

C caudally directed influence excites start of return stroke in the posterior leg
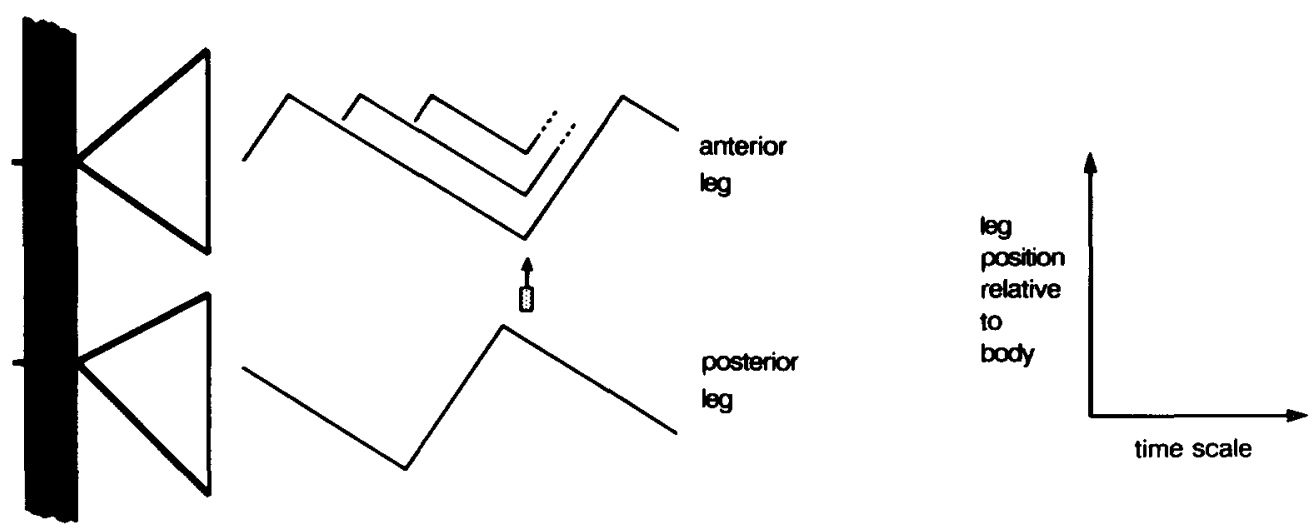

B rostrally directed influence

excites start of return stroke in the anterior leg

Fig. 4. Coordination between two ipsilateral legs of a stick insect. Upper traces show the anterior leg. Each schema is drawn as if only one of the three coordinating mechanisms existed. In each case the influencing leg is drawn only once. For the influenced leg several traces are presented to show the effect of the coordinating mechanism. The duration and the intensity of the influences are roughly indicated by the length and the thickness of the bars and wedges, respectively. (A) The rostrally directed inhibitory influence is active during the return stroke of the posterior leg. (B) The rostrally directed excitatory influence is active at the beginning of the power stroke of the posterior leg. (C) The caudally directed excitatory influence is active during the power stroke of the anterior leg. The terms 'excitatory' and 'inhibitory' are applied according to the effect on the start of the return stroke in the influenced leg. The duration of the return stroke could not be shown to be influenced by these coordinating mechanisms. As it is not known in detail how the varying amplitudes are compensated, return strokes are only roughly indicated. (Modified from Ref. 14.)

the ipsilateral mechanism where the influence is only active during a small fraction of the whole step cycle (see Fig. 2B), the contralateral influence continues during most of the cycle (Fig. 3). With the exception of a small phase range the return stroke of the other leg is always shortened. Contralateral coupling is much weaker than ipsilateral coupling. While in ipsilateral legs the effect of a disturbance is compensated within the following step, because of the weaker coupling in contralateral legs a disturbance results in a so-called 'gliding' coordination (the 'relative' coordination of von $\mathrm{Holst}^{17}$ ). This means that several steps are necessary to regain normal coordination. Contralateral coupling acts in the same way in both directions but one leg might be slightly more dominant. This dominance seems to be inherent to some extent but can also be influenced experimentally by applying different loads to the legs.

\section{Coupling mechanisms in stick insects}

Quite different results were found concerning the coordinating mechanisms in the stick insect. Three different mechanisms influence the PEP, i.e. the transition from power stroke to return stroke. They are shown schematically in Fig. 4. Two influences are rostrally directed. One (Fig. 4A) hinders a leg from starting a return stroke while the posterior leg performs its return stroke and may continue for about another $100 \mathrm{~ms}$. This influence can prolong the power stroke $\mathrm{e}^{14,18,19}$. Experimental results indicate that this is done by shifting the threshold for beginning a return stroke to a more posterior position ${ }^{9}$. In contrast to this 'inhibitory' influence the two other mechanisms have 'excitatory' effects. The second rostrally directed influence (Fig. 4B) can elicit a return stroke in a leg when the posterior leg starts a power stroke $^{14}$. This influence shortens the duration of the power stroke. A third influence is caudally directed (Fig. 4C): the start of a return stroke occurs earlier, the farther the anterior leg is moved rearward during its power stroke ${ }^{14,19-21}$. This causes the posterior leg to perform a return stroke before the anterior leg begins its return stroke. These three mechanisms are redundant in that they produce the same effect - the nearly immediate re-establishment of coordination in the case of any disturbance. 
In addition, two other, less important, coordinating mechanisms are found in the stick insect. During walking the hind leg tarsus is placed immediately behind the middle leg tarsus ${ }^{22,23}$. The same 'targeting' occurs between middle and front leg and also between front leg and antennae (Cruse, H., unpublished observations). The main function of these mechanisms is presumably to help a leg find ground contact, an essential function when climbing on branches, but trivial when walking on a continuous surface. Another coupling mechanism is the so-called 'co-activating' influence, which affects the motor output during the power stroke ${ }^{24,25}$. An increase in the motor output of a leg immediately leads to an increase in the motor output of neighbouring legs during their power stroke. Thus, these co-activating mechanisms provide an increase in the propulsive force of the whole animal. In contrast to the influences discussed above, the coactivating influence is a mutual influence between all immediately adjacent legs with the exception of the two hind legs.

These co-activating influences are not the only contralateral effects. As was found for the crayfish, coupling between contralateral legs is weaker than that between ipsilateral legs. However, two of the three mechanisms found for ipsilateral legs also act between contralateral legs of the same segment. These are the two 'excitatory' mechanisms shown in Fig. 4B, C. As in the crayfish these contralateral influences seem to act in both directions between the two legs of one segment.

\section{The neuronal basis of coordination}

On the neuronal level a lot is known about the control of an individual leg. However, little information is available concerning the problem of coordination between legs (for reviews see Refs 1, 26, 27). The electrophysiological data are mostly qualitative in the sense that no detailed phase dependencies were measured and they therefore cannot yet be compared quantitatively with the behavioural data. Within these limitations there is a correspondence between the results obtained by both methods: in crustacea an alternating rostrally directed influence was found that might correspond to that shown in Fig. $2 \mathrm{~A}^{28}$. In stick insects the hind legs can be driven experimentally in the rhythm of the middle legs, implying a caudally directed influence ${ }^{29}$. When the front legs were intact but meso- and metathoracic ganglia were completely deafferented, the activity of protractor motoneurons of the middle leg was phase-dependent in that its activity increased during the course of the front leg retraction (Fig. 5). This was also found to occur between intact middle and deafferented hind legs ${ }^{21}$. Both effects probably correspond to the caudally directed mechanism described in Fig. 4C. In the cockroach a mutual coupling of ipsilateral legs was found that inhibits a burst of flexor motoneurons in one leg during a flexor burst in the neighbouring $\mathrm{leg}^{30}$. The flexor burst corresponds to a return stroke in a walking animal. Thus it might correspond to the mechanism shown in Fig. $4 \mathrm{~A}$ which, in contrast to the behavioural results obtained in stick insects, would act in both directions. But as in these experiments the animals were pinned upside down (and deafferented) it is doubtful whether these results can actually be attributed to walking activity ${ }^{31,32}$. By simultaneously measuring leg movement and electrophysiological activity in the stick insect, it was found that during a prolonged power stroke of the hind leg, changes linked to the movement of the middle leg can be variable (Fig. 6). They can be (1) weak and only decrease the frequency of action potentials in the hind leg power stroke, or (2) stronger and stop the power stroke activity to start a short return stroke burst producing a short lifting of the leg, or (3) strong enough to elicit a real return stroke. It is not clear whether this results from a changing intensity of the signal from the middle leg or whether it represents a changing sensitivity of the hind leg pattern generator to coordinating signals. However, the results show that the coordination mechanisms known so far do not influence only the timing of the leg's pattern generator but also possibly the strength of excitation at any given position (see Ref. 33 for review). Individual neurons that might transmit the coordinating signals between ganglia have been described in locusts ${ }^{34,35}$. It is, however, not yet possible to ascribe a meaning to them in the functional context discussed here.

A first step towards locating the coordinating mechanisms on a more neuronal level than by directed electrophysiological recordings is to cut connectives. Results of such experiments in the cockroach $^{36}$ suggest that contralateral influences exist between the front legs and between the hind legs, but no work has been done on the coupling between middle legs. Furthermore, after cutting a connective the two adjacent legs start some return strokes during the return stroke of the other leg, indicating that an inhibitory coupling mechanism is destroyed. A detailed investigation of cutting connectives in the stick insect showed that all known coordinating mechanisms seem to be conveyed by the ipsilateral connective ${ }^{37}$.

\section{Height control}

The coordination of legs in a walking animal is discussed here only with respect to the movement in the forward-rearward direction, i.e. the horizontal component of the movement. However, when walking over an uneven surface, the legs have to act together in order to control the height of the body and coordinate movement in the vertical direction. The behaviour of a stick insect walking over different obstacles $^{38}$ or standing on different substrata ${ }^{39}$ can be quantitatively described according to a hypothesis that each leg acts as a vertical 'spring' which is independent of the other legs; thus the mechanical connections via the body are sufficient and no neuronal coupling between the legs is necessary to control body height. 
Fig. 6. Recording from protractor and/or retractor coxae nerves of the right middle and hind leg of the stick insect Carausius morosus. All legs except the right hind leg walk on a treadwheel. The right hind leg walks on a motor-driven belt with a slower speed. Therefore the power stroke of the hind leg is about three times longer than that of the middle leg.

During the power stroke of the hindleg the hind leg protractor might be excited, which could lead to a slight lifting of the tarsus (star) or a full return stroke. (Modified from Ref. 20.)

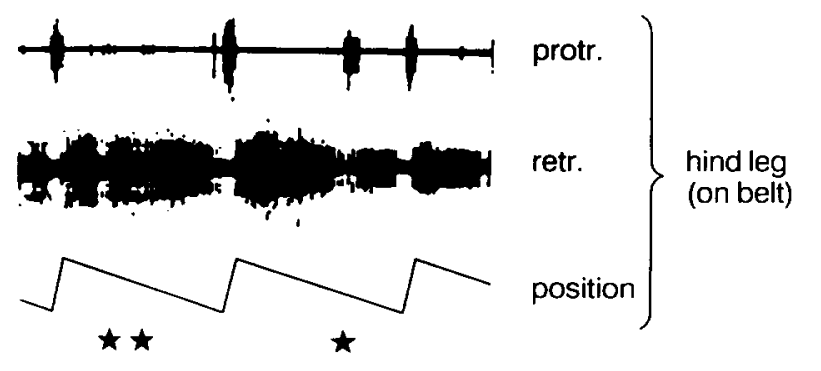

(on wheel)

\section{Control exerted by other neural centres}

An animal does not always walk. Therefore it has to be possible to activate and inactivate the neuronal circuitry that produces walking movements. This circuitry can be imagined to consist of systems like that schematically represented by Fig. 1B, which has to be completed by coordinating influences. An animal can start walking spontaneously or by being excited by external, e.g. tactile, stimuli. Some crustacea were found to be easily stimulated to walk when the legs of the animal were moved rearwards by moving the ground beneath the animal using a motor-driven belt. Bässler ${ }^{7}$ found that in the stick insect, even when the sense organ of a single joint, the femoral chordotonal organ, signals a rearward movement, the neural circuitry of the leg can be excited to start walking, if the central excitation of the animal is high enough. The same organ is shown to be a feedback transducer in the velocity feedback loop. Thus, the stimulus 'movement of the leg' affects the system on different levels, on a lower level as a velocity feedback and on a higher level activating the whole system (the latter is not shown in Fig. 1B).

When the standing stick insect is stimulated to walk by, for example, a tactile stimulus, all legs that are on the ground start with caudally directed forces ${ }^{40}$. This shows that the neural circuitry of each leg always starts in the state of power stroke when activated after a pause. This is, however, not generally the case. Land ${ }^{11}$ reported that in the jumping spider, a leg can also stop the movement within a return stroke and later continue walking by completing the interrupted return stroke. Influences on the leg pattern generators are also necessary when the animal walks in curves. As reviewed by Graham ${ }^{1}$, examples for both theoretical possibilities can be found: (1) the coordination between right and left legs can be maintained and the step amplitude, the distance between AEP and PEP, increases for the legs on the outside and decreases for those on the inside of the curve; (2) right and left legs can become uncoupled and the outside legs walk with higher frequency.

\section{Concluding remarks}

The results, which up to now have been mainly based on behavioural data, show that each walking leg can be considered as an oscillating unit with the properties of a relaxation oscillator. While the internal organization of this oscillator seems to reflect a hierarchical structure, the coupling between the different oscillators is organized in a non-hierarchical manner. The coupling between two such oscillators is not continuous as is the case in many mathematical descriptions of coupled oscillators. In contrast, the coupling between walking legs seems to be active only within selected phase ranges and 'switched off' at other times. Ipsilateral influences are asymmetric in the sense that rostrally directed influences are in general qualitatively different to caudally directed ones. Contralateral mechanisms seem to be symmetrical although quantitative differences can be found such that one side is able to dominate the other to some extent. There is a redundancy in the sense that different mechanisms produce basically the same result, namely a proper coordination between legs. The existence of several different mechanisms provides the possibility of a faster recovery of normal coordination after a disturbance, and makes the whole system more stable. Earlier theories involving coupling mechanisms that acted only in one direction had to assume the existence of a hierarchy of 'eigenfrequencies' in the different oscillators. This means, for example, that the front legs if uninfluenced by coordinating signals walked faster than middle legs and these in turn walked faster than hind legs (see Ref. 41 for review). Because of the combination of different caudally and rostrally directed coupling mechanisms this assumption is no longer necessary.

The mechanisms seem to be quite different in different animals. Comparative studies showed that the mechanisms in the crayfish and in the rock lobster are very similar ${ }^{42}$. Forward-directed inhibitory influences were found for the stick insect, for the grasshopper ${ }^{1}$ and probably exist in the cockroach. One can speculate that the differences between crayfish and stick insects reflect those between decapods and insects. Whether this is so or not, one might look for ecological causes of these differences. What might be the reason for the different strategies used for the coordination of legs in crayfish and in stick insects? A basic difference is that maintaining the stability of the body position is much less a problem for the crayfish walking under water and using eight legs than for the stick insect walking on land and climbing on branches with only six legs. Therefore the stick insect is under much more constraint to keep as many legs on the ground as possible at one time. It may be that in stick insects coordination has to be obtained by a shorter or longer prolongation of the power stroke, whereas the crayfish can afford to solve the coordination problem by maintaining legs in the return stroke for longer. The latter might be the simpler computational task because (1) stability problems that arise when changing the end of the return stroke might generally be less critical than those that arise when changing the end of the power stroke and (2) the control of the leg movement is easier during the return stroke when the movement of the leg is not mechanically coupled to the other legs.

\section{Selected references}

1 Graham, D. (1985) Adv. Insect Physiol. 18, 31-140

2 Cruse, H. and Pflüger, H-J. (1981) J. Exp. Biol. 92, 97-107

3 Dean, J. (1984) J. Comp. Physiol. A 155, 771-781

4 Cruse, H. (1985) J. Exp. Biol. 116, 343-355

5 Schmitz, J. (1985) in Insect Locomotion (Gewecke, M. and 
Wendler, G., eds), pp. 27-32, Parey

6 Weiland, G. and Koch, U. T. (1987) J. Exp. Biol. 133 137-156

7 Bässler, U. (1988) J. Exp. Biol. 136, 125-147

8 Bässler, U. (1977) Biol. Cybern. 25, 61-72

9 Cruse, H. (1985) J. Exp. Biol. 116, 357-362

10 Cruse, H. (1985) in Insect Locomotion (Gewecke, M. and Wendler, G., eds), pp. 19-26, Parey

11 Land, M. F. (1972) J. Exp. Biol. 57, 15-40

12 Bässler, U. (1986) Biol. Cybern. 54, 65-69

13 Cruse, $H$. and Müller, U. (1986) J. Exp. Biol. 121, 349-369

14 Cruse, H. and Schwarze, W. (1988) I. Exp. Biol. 138 455-469

15 Cruse, H. and Knauth, A. (1989) J. Exp. Biol. 145, 199-213

16 Warnecke, H. and Cruse, H. (1989) Verh. Dtsch. Zool. Ges. 81,148

17 von Holst, E. (1943) Pflügers Arch. Ges. Physiol. 246, 846-865

18 Cruse, H. and Epstein, S. (1982) J. Exp. Biol. 101, 161-170

19 Dean, J. and Wendler, G. (1982) J. Comp. Physiol. 148, 195-207

20 Foth, E. and Bässler, U. (1985) Biol. Cybern. 51, 313-318

21 Bässler, U. Dübner, C. and Fahrig, T. (1987) Zool. Jahrb. Physiol. 91, 393-401

22 Cruse, H. (1979) Physiol. Entomol. 4, 121-124

23 Dean, J. and Wendler, G. (1983) J. Exp. Biol. 103, 75-94

24 Bässler, U. (1979) Physiol. Entomol, 4, 193-199

25 Cruse, H. (1985) J. Exp. Biol. 114, 513-519
26 Bässler, U. (1983) Neural Basis of Elementary Behavior in Stick Insects Springer-Verlag

27 Clarac, F. (1984) Trends Neurosci. 7, 293-298

28 Clarac, F. and Barnes, W. J. P. (1985) in Coordination of Motor Behaviour (Bush, B. M. H. and Clarac, F., eds), pp. 249-269, Cambridge University Press

29 Bässler, U. and Wegner, U. (1983) J. Exp. Biol. 105, 127-145

30 Pearson, K. G. and lles, F. J. (1973) J. Exp. Biol. 58, 725-744

31 Reingold, S. C. and Camhi, J. M. (1977) J. Insect Physiol. 23, 1407-1420

32 Zill, S. N. (1986) J. Neurobiol. 17, 317-328

33 Bässler, U. (1987) Biol. Cybern. 55, 397-401

34 Laurent, G. and Burrows, M. (1988) J. Comp. Neurol. 275, $1-12$

35 Ritzmann, R. E. and Pollack, A. J. (1988) J. Neurobiol. 19 $589-611$

36 Greene, S. and Spirito, C. (1979) J. Exp. Biol. 78, 245-253

37 Dean, J. J. Exp. Biol. (in press)

38 Cruse, H. (1976) Biol. Cybern. 24, 25-33

39 Cruse, H., Riemenschneider, D. and Stammer, W. (1989) Biol. Cybern. 61, 71-77

40 Cruse, H. and Saxler, G. (1980) Biol. Cybern. 36, 159-163

41 Cruse, H. and Graham, D. (1985) in Coordination of Motor Behaviour (Bush, B. M. H. and Clarac, F., eds), pp. 283-301, Cambridge University Press

42 Clarac, F. (1985) in Feedback and Motor Control in Invertebrates and Vertebrates (Barnes, W. J. P. and Gladden, M. H., eds), pp. 379-400, Croom Helm

\title{
Cognitive function in Parkinson's disease: from description to theory
}

\author{
R. G. Brown and C. D. Marsden
}

From the large body of empirical evidence on cognitive function in Parkinson's disease, a number of attempts have been made to describe the characteristics of the deficits and the conditions under which they are observed. This review considers descriptions limited to specific domains of cognition such as visuospatial function, memory and 'frontal' function, and more general descriptions relating to 'set-shifting', sequencing, temporal ordering and recency discrimination, the locus of cognitive control and bradyphrenia. Later in the paper an attempt is made to provide some theoretical framework for the various descriptions. Two theories are discussed representing contrasting, but complementary approaches. The first is a 'psychological' theory in which the concept of depleted processing resources is suggested as a possible mechanism to explain the observable deficits. The second is a neurobiological model that attempts to integrate information from diverse sources to provide a model for the neuroanatomical and neurochemical substrate that may underlie some of the behavioural deficits.

The number of publications on cognition in Parkinson's disease (PD) has grown exponentially in the past three decades. This reflects not only great interest in this aspect of PD itself, but also the belief that the cognitive abnormalities may give some clue as to normal cognitive function. In particular, the studies may hint at the role of the basal ganglia and their cortical connections in cognition. The research can be considered at three levels. First, particularly in the early days, research took a pragmatic 'look and see' approach, in which individual tests or groups of tests were administered to largely unselected groups of subjects. From this research, certain patterns of impaired and intact performance became evident. The second stage has been an attempt to define these deficits more precisely, the conditions under which they occur, and occasionally, whether particular subgroups of patients are more susceptible. These studies, although guided by previous empirical evidence, have been largely descriptive (see Box 1$)^{1-7}$. Some have been limited to deficits affecting a single domain of cognitive function such as memory or visuospatial function, or an anatomically defined region such as in the 'frontal' deficit. Others have sought to define a broader range of deficits that cross the classic boundaries of neuropsychological function, including bradyphrenia, 'set-shifting', internal attentional control, deficits in sequencing, temporal organization and recency discrimination. These two categories should not be seen as mutually exclusive. In some cases they overlap, while in others they may be hierarchically organized. It should be noted that this paper does not aim to be an exhaustive review of the literature on cognitive function in PD. One major area not included in the present article is that of dementia in PD and its relationship to other neurodegenerative disorders. The reader is referred to a recent review that covers this area in detail ${ }^{8}$.

\section{Descriptions of cognitive function in Parkinson's disease}

The visuospatial deficit. An earlier review ${ }^{9}$, considered the proposal that patients with $\mathrm{PD}$ had a generalized visuospatial deficit. A number of studies
R. G. Brown and C. D. Marsden are at the MRC Human Movement and Balance Unit, National Hospital, and Department of Clinical Neurology, Institute of Neurology, Queen Square, London WC1N 3BG, UK. 